\title{
Comparison of Black Bone MRI and 3D-CT in the preoperative evaluation of patients with craniosynostosis
}

\author{
Anne Saarikko ${ }^{a}$, Eero Mellanen ${ }^{\mathrm{a}, *}$, Linda Kuusela ${ }^{\mathrm{b}, \mathrm{c}}$, \\ Junnu Leikola ${ }^{a}$, Atte Karppinen ${ }^{\mathrm{d}}$, Taina Autti ${ }^{\mathrm{e}}$, \\ Pekka Virtanen ${ }^{\mathrm{e}}$, Nina Brandstack ${ }^{\mathrm{e}}$
}

\author{
${ }^{a}$ Cleft Palate and Craniofacial Centre, Department of Plastic Surgery, Helsinki University Central \\ Hospital, Topeliuksenkatu 3-5, PO Box 266, 00029 Helsinki, Finland \\ ${ }^{\mathrm{b}}$ HUS Medical Imaging Center, Helsinki University Hospital, Helsinki, Finland \\ ' Department of Physics, University of Helsinki, Finland \\ d Department of Neurosurgery, Helsinki University and Helsinki University Hospital, Helsinki, Finland

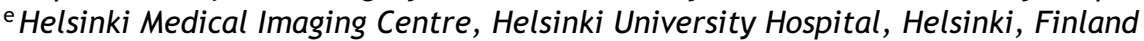

Received 20 December 2018; accepted 22 November 2019

Available online $x x x$

\section{KEYWORDS}

Pediatric Imaging;

Scaphocephaly;

Craniofacial surgery;

Syndromatic

craniosynostosis;

Chiari malformation

\begin{abstract}
Summary Purpose: Black Bone (BB) magnetic resonance imaging (MRI) is a nonionizing imaging method and a recent alternative to computed tomography (CT) in the examination of cranial deformities. The purpose of this study was to compare BB-MRI and routine 3D-CT in the preoperative evaluation of patients with craniosynostosis.

Methods: At our center, we have routinely performed preoperative CT of the skull and brain MRI for patients with clinical suspicion of craniosynostosis. We recently changed our MRI protocol into one that includes sequences for the evaluation of both brain anatomy and skull bone and sutures by BB-MRI. A semi-automatic skull segmentation algorithm was developed to facilitate visualization. Both BB-MRI and 3D-CT were performed on 9 patients with clinical craniosynostosis, and the images were evaluated by two craniofacial surgeons, one pediatric neurosurgeon, and two neuroradiologists.

Results: We obtained informative 3D images using BB-MRI. Six (6/9) patients had scaphocephaly, 1 (1/9) patient had unicoronal synostosis, and 2 (2/9) patients had lambdoid synostosis. The affected synostotic sutures could be identified both by BB-MRI and by 3D-CT in all patients. Intra-rater and inter-rater reliability for rating the calvarial sutures was high. However, the reliability for rating the intracranial impressions was low by both imaging methods.
\end{abstract}

* Corresponding author.

E-mail address: eero.mellanen@helsinki.fi (E. Mellanen). 
Conclusion: $\mathrm{BB}-\mathrm{MRI}$ is an alternative to $3 \mathrm{D}-\mathrm{CT}$ in the preoperative evaluation of patients with craniosynostosis. BB-MRI provides information not only on cranial sutures and intracranial impressions but also on the brain structure in one imaging session. This method can replace ionizing radiation-based methods in analyzing skull deformities.

(C) 2019 British Association of Plastic, Reconstructive and Aesthetic Surgeons. Published by Elsevier Ltd. All rights reserved.

\section{Introduction}

The coronal, sagittal, lambdoid, and squamosal sutures normally fuse around the fourth decade of life; the metopic suture fuses within the first year of life. ${ }^{1}$ Craniosynostosis is defined as the premature fusion of one or more of the sutures between the cranial vault bones. ${ }^{2,3}$ These premature fusions can be further classified into categories depending on whether they appear as a part of a syndrome (syndromatic) or as an isolated defect (non-syndromatic). ${ }^{2,3}$ The relationship between premature calvarian suture fusion and head shape anomalies in craniosynostosis is established. ${ }^{3}$ Craniosynostosis is a relatively rare malformation, and its prevalence ranges from 2.6 to 7.2 per 10,000 live births. ${ }^{4-6}$ The most common non-syndromatic single-suture craniosynostosis is sagittal synostosis, which occurs in $40 \%$ to $60 \%$ of cases. $^{4-7}$

The diagnosis of craniosynostosis is achieved by physical examination and medical imaging. ${ }^{2,8}$ In most cases, the shape of the skull reveals the underlying synostosis. Diagnostic imaging is justified for the evaluation of the patency of the remaining sutures and possible intracranial pathology (including Chiari malformation) and to aid in planning the surgical procedure. ${ }^{2,8-11}$ Imaging may reveal signs of diffuse intracranial impressions as a potential indicator of increased intracranial pressure (ICP). ${ }^{8}$ In patients with craniosynostosis, imaging has relied on low-dose computed tomography (CT) and on the subsequently constructed 3D images. Despite the unavoidable exposure to ionizing radiation and the well-known risks associated with $\mathrm{CT},{ }^{12-16}$ low-dose CT is currently the imaging technique of choice for patients with craniosynostosis.

Children are more sensitive to ionizing radiation than adults. ${ }^{12,13,17}$ Thus, whenever available, a non-ionizing imaging modality should be the imaging method of choice. The limitation of MRI has been its poor ability to show bony details. The role of MRI in the diagnostic evaluation of children with skull deformities has thus been minimal. In 2012, Eley et al. presented a novel Black-Bone MRI (BB-MRI) sequence that significantly improved the contrast between soft tissue and the bone and allowed visualization of the calvarian bone, sutures, and craniofacial skeleton. ${ }^{18-21}$ We introduced the BB-MRI method first for the diagnostic imaging of patients with posterior plagiocephaly along with neurological symptoms. ${ }^{22}$

The purpose of this study was to compare BB-MRI and $\mathrm{CT}$ in the preoperative evaluation of patients with craniosynostosis. 3D-reconstructed images of the cranial vault were created from the data obtained from the two imaging modalities, and the appearance of the cranial sutures was assessed. We also evaluated the quality of the 3D reconstructions created with BB-MRI and compared them with that of the $3 \mathrm{D}$ reconstructions presented in the literature. Our aim was to provide high-quality 3D-reconstructed images of the cranial vault for routine clinical practice by a nonionizing imaging method.

\section{Patients and methods}

Ethical approval for the use of BB-MRI was granted by the Helsinki University Hospital Research Ethics Committee. It is a routine practice at our craniofacial center to perform MRI of the brain as a preoperative evaluation of potential intracranial findings (such as Chiari malformation) for all patients with craniosynostosis. From 2016 to 2018, a total of 9 children with clinical suspicion of craniosynostosis underwent 3D-CT and an MRI protocol that included sequences for the evaluation of both brain anatomy and skull bone and sutures by BB-MRI. The accuracy and diagnostic value of BB-MRI to confirm the diagnosis of craniosynostosis and to visualize synostotic or patent sutures and intracranial impressions were assessed by comparing the imaging data received by $3 \mathrm{D}-\mathrm{CT}$ and $\mathrm{BB}-\mathrm{MRI}$. In this study population, $B B-M R I$ imaging was performed in the same imaging session with routine brain MRI.

MRI was performed with a Siemens Skyra 3T (Erlangen, Germany) with a 32-channel head coil. Two MRI protocols were set up for imaging depending on patient age and need for anesthesia. The feed-and-sleep method was used for patients aged under 6 months and general anesthesia for others. Patients P2, P4, and P5 were imaged using the bottle-fed protocol, which utilizes Siemens Quiet Suite sequences and consists of an in-phase Blackbone StarVibe sequence and T2-weighted quiet BLADE coronal and axial sequences. The StarVibe sequence employs a stack-of-stars k-space acquisition, which is less motion-sensitive than the VIBE sequence. ${ }^{23}$ The remaining patients were imaged under general anesthesia, and the imaging protocol is presented in Kuusela et al. ${ }^{22}$ The image segmentation for the Blackbone Starvibe sequence was performed by altering the algorithm presented in Kuusela et al. to use only the in-phase image. ${ }^{22}$ For the StarVibe sequence, large parts of the skull had to be segmented manually. The development of the sequence and algorithm is ongoing.

\section{Image analyses}

Radiological evaluation of the structural and BB-MRI sequences was performed by two neuroradiologists (NB and $P V)$. Information on the cranial sutures and intracranial impressions received from 3D-CT and BB-MRI was evaluated by two craniofacial surgeons (JL and AS), one pediatric 
Table 1 Patient summary.

\begin{tabular}{lllll}
\hline Patient ID & Diagnosis/synostosis & Sex & Age in CT (M) & Age in BB-MRI (M) \\
\hline P1 & Unicoronal synostosis, right & $\mathrm{F}$ & 6.2 & 7.9 \\
P2 & Sagittal synostosis & M & 5.6 & 4.9 \\
P3 & Sagittal synostosis & M & 2.8 & 2.6 \\
P4 & Sagittal synostosis & $\mathrm{F}$ & 55.9 & 59.9 \\
P5 & Sagittal synostosis & M & 5.1 & 4.1 \\
P6 & Unilamboid synostosis, left & $\mathrm{F}$ & 1.6 & 7.5 \\
P7 & Unilamboid synostosis, left & $\mathrm{F}$ & 76.2 & 78.9 \\
P8 & Sagittal synostosis & $\mathrm{F}$ & 5 & 5.2 \\
P9 & Sagittal synostosis & M & 91.6 & 94.8 \\
\hline
\end{tabular}

neurosurgeon (AK), and two neuroradiologists (NB and PV). Inter-rater reliability between raters was calculated. The two neuroradiologists performed the evaluation blinded and repeated the evaluation after 2 weeks to evaluate intrarater reliability. Five (sagittal, right coronal, left coronal, right lambdoid, and left lambdoid) sutures of each patient were included in the analyses. Anterior and posterior sagittal sutures were rated separately. Each of the sutures was rated to be open, partially fused, or completely fused. Impressions were evaluated in three different locations of the skull: anterior, parietal, and posterior (see Table 3). In each location, intracranial impressions were graded as severe (2), mild (1), or absent (0).

\section{Statistical analyses}

Intra-rater reliability was calculated with both percent agreement and Cohen's $\kappa$ between ratings. The interrater reliability among the five raters was calculated with Krippendorff's alpha. The analyses were performed using Microsoft Excel and SPSS version 24.

\section{Results}

This study material consisted of 9 patients ( 5 females and 4 males) with clinical diagnosis of craniosynostosis and clinical indication for preoperative CT and MRI. Six (6/9) patients had scaphocephaly, 1 (1/9) patient had unicoronal synostosis, and 2 (2/9) patients had lambdoid synostosis. The diagnosis of all patients was confirmed by routine 3D-CT. The average age at the time of CT was 27.8 (range 1.6-91.6) months and that at the time of BB-MRI was 29.5 (range 2.6-94.8) months (see Table 1).

We obtained informative 3D images of the skull bone and sutures using BB-MRI (Figures 1-4). The skull segmentation was based on bias field-corrected fuzzy c-means clustering, ${ }^{24}$ which aims to calculate the probabilities of belonging to a cluster. This requires that the surrounding of a voxel consists of similar types of voxels to be classified as belonging to a cluster. The slice thickness of the BB-MRI sequence was $1 \mathrm{~mm}$, and the skull of the infants was approximately the same thickness. Thus, classifying the clusters correctly in infants with a thin skull was more challenging.

The imaging data obtained by routine 3D-CT and BB-MRI were compared by all reviewers (see summary in Table 2).
The same diagnosis of craniosynostosis was made for 9 patients by all five reviewers. While the calvarian sutures were also visualized from the BB-MRI images in the youngest (2-5 months) patients (see Figure 4), the visualization of the bony structures was more accurate in the older patients. In patient P8 with sagittal synostosis, the right coronal suture was rated partially synostotic in BB-MRI by the pediatric neurosurgeon. In patient P4 with sagittal synostosis, the right lambdoid suture was rated to be partially synostotic by one of our neuroradiologists. The inter-rater reliability for evaluating the sutures by routine 3D-CT and BB-MRI was Kalpha 0.953 and Kalpha 0.950 , respectively. The intrarater reliability between ratings by routine $3 \mathrm{D}-\mathrm{CT}$ with percent agreement was 0.963 (neuroradiologist 1) and 1.00 (neuroradiologist 2 ). The Cohen's $\kappa$ reliability was found to be $\kappa=0.914$ (neuroradiologist 1) and $\kappa=1.00$ (neuroradiologist 2). The intra-rater reliability between ratings by BB-MRI with percent agreement was 0.907 (neuroradiologist 1) and 0.870 (neuroradiologist 2 ). The Cohen's $\kappa$ reliability was $\kappa=0.8$ (neuroradiologist 1 ) and $\kappa=0.731$ (neuroradiologist 2).

Intracranial impressions were evaluated by the reviewers in 3D-CT and BB-MRI; the results are summarized in Table 3. A variable amount of intracranial impressions was detected in the skull bone in all 9 patients. There was some variation in how impressions were visualized by 3D-CT in comparison to BB-MRI (Table 3). The impressions were typically rated more intense in BB-MRI than in 3D-CT $(5 / 27)$. In one patient, $1 / 27$ impressions were rated more intense in 3D-CT than in BB-MRI. In most comparisons, the impressions were evaluated similarly in BB-MRI and 3D-CT (21/27). Inter-rater reliability for the assessment of impressions was low by both methods (3D-CT, Kalpha 0.553; BB-MRI, Kalpha 0.458 ). Intra-rater reliability between ratings by routine 3D-CT with percent agreement was 0.667 (neuroradiologist 1) and 0.778 (neuroradiologist 2). The Cohen's $\kappa$ reliability was found to be $\kappa=0.461$ (neuroradiologist 1) and $\kappa=0.634$ (neuroradiologist 2). Intra-rater reliability between ratings by BB-MRI with percent agreement was 0.778 (neuroradiologist 1) and 0.667 (neuroradiologist 2). The Cohen's $\kappa$ reliability was $\kappa=0.62$ (neuroradiologist 1 ) and $\kappa=0.491$ (neuroradiologist 2).

In our study, no structural malformations of the brain were observed in any patient. Patient $\mathrm{P} 8$ had two very small left cerebellar T2 dark signal foci in a location typical for perinatal germinal matrix hemorrhage. The visualization of the bony structures with BB-MRI was found to be more 


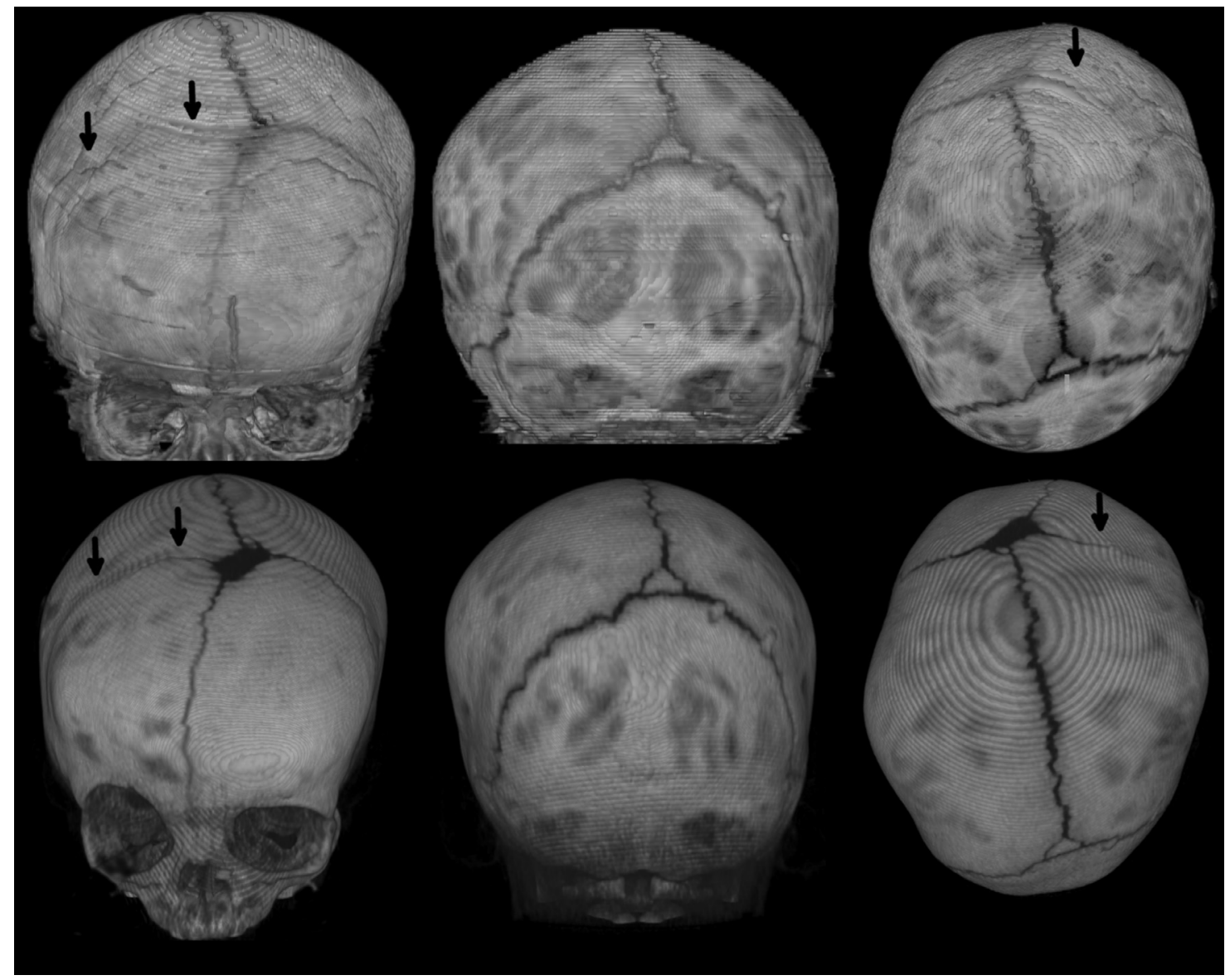

Figure 1 Skull images of patient 1 with right coronal synostosis. Top row: 3D-rendered BB-MRIs at the age of 7 months. Bottom row: 3D-rendered CT images of the same patient at the age of 6 months. The arrows point to the location of the fused suture.

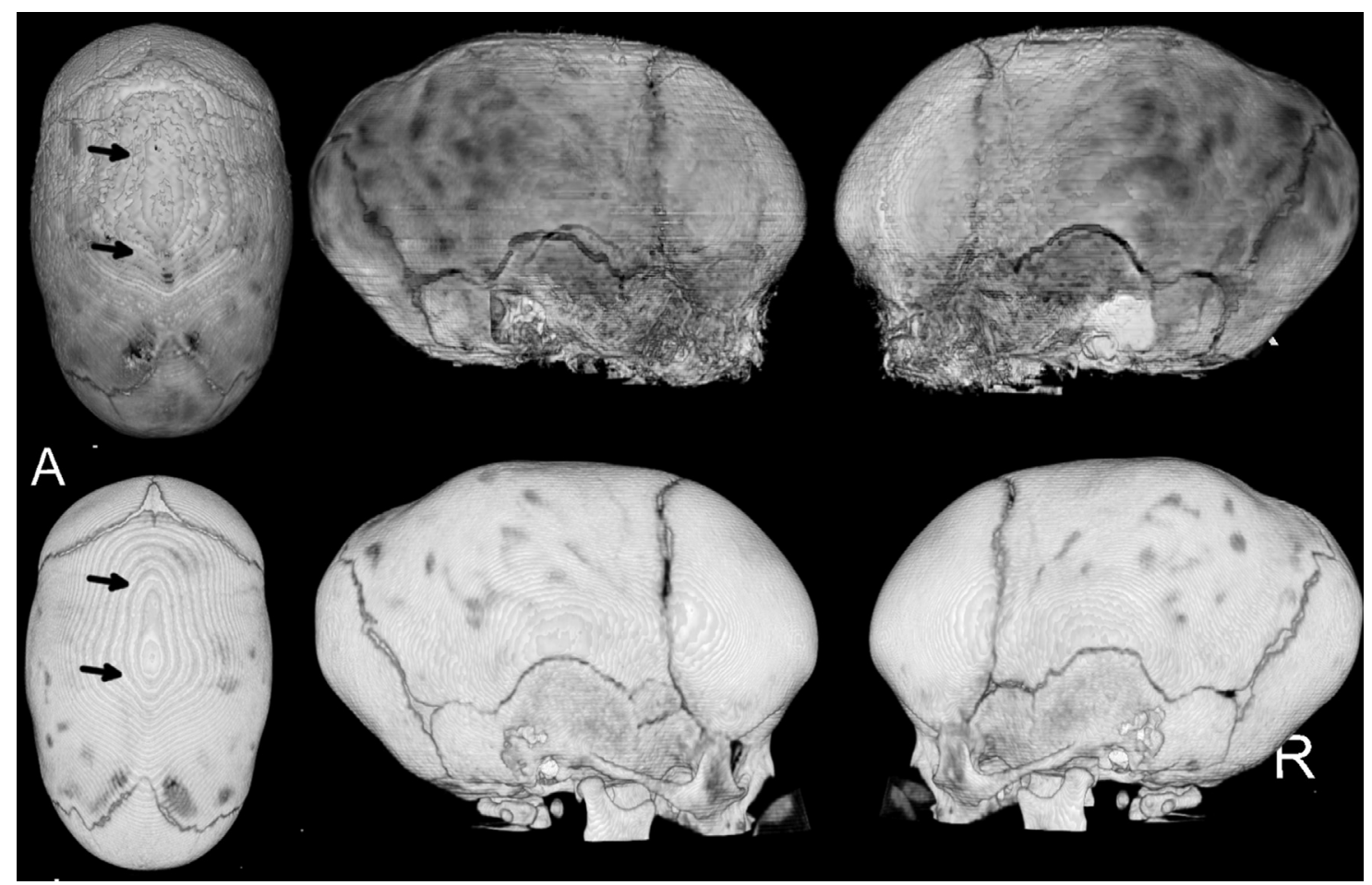

Figure 2 Skull images of patient 5 with sagittal synostosis. Top row: 3D-rendered BB-MRIs at the age of 4 months. Bottom row: 3D-rendered CT images at the age of 5 months. The arrows point to the location of the fused suture. 


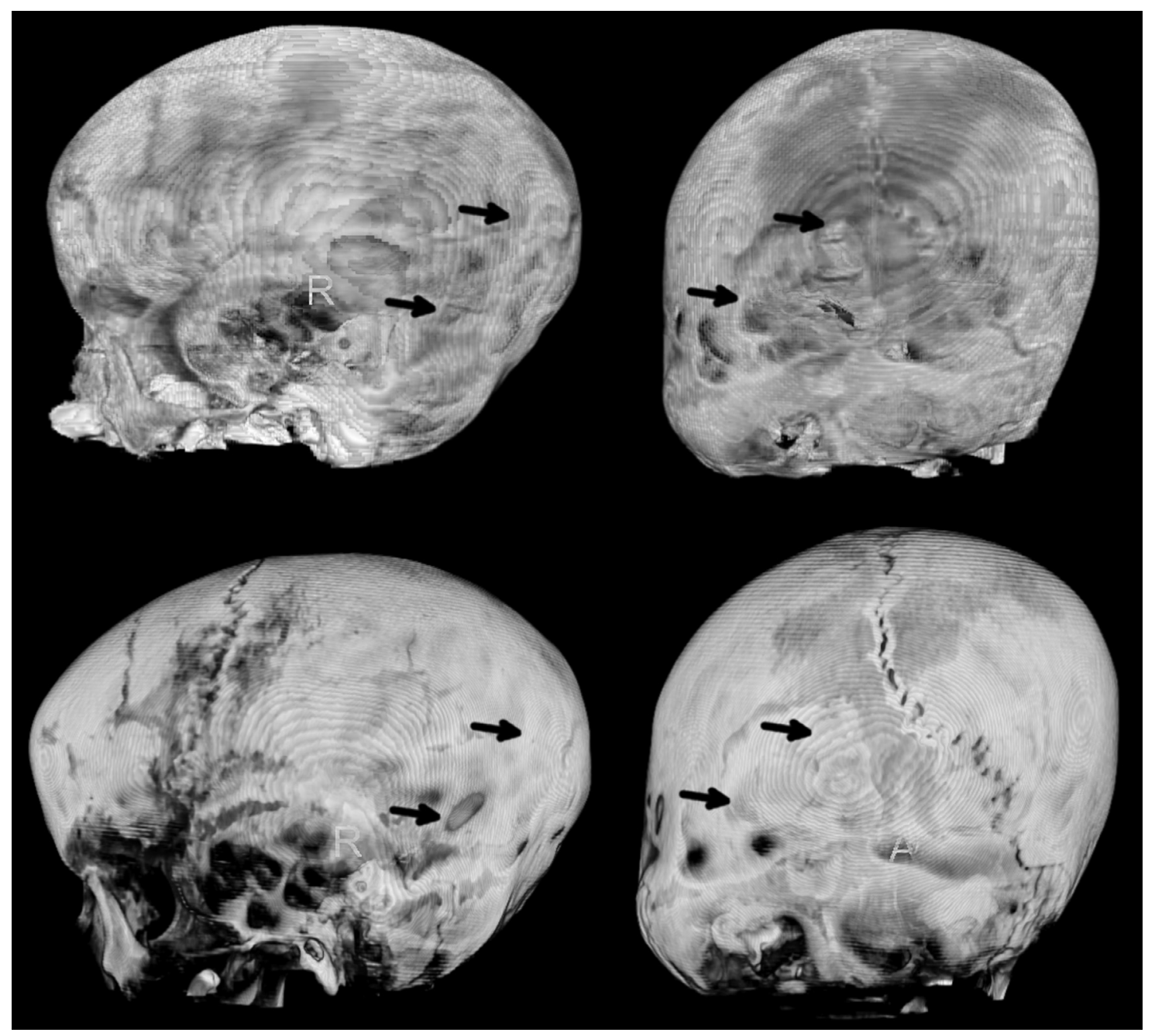

Figure 3 Skull images of patient 7 with left lambdoid synostosis. Top row: 3D-rendered BB-MRIs at the age of 78 months. Bottom row: 3D-rendered CT images at the age of 76 months. The arrows point to the location of the fused suture.

accurate in older patients than in patients aged under 6 months.

\section{Discussion}

The aim of this study was to assess BB-MRI in the preoperative evaluation of patients with craniosynostosis. The BB-MRI method presented by Eley et al. ${ }^{19}$ was used and further developed as a suitable MRI protocol for diagnostic imaging of patients with craniosynostosis.

In routine diagnostic evaluation of children with skull deformities, CT allows documentation of the patency or closure of the sutures and aids in surgical planning. ${ }^{2,8}$ Most cranial sutures are best assessed using 3D reconstruction of images, as these images provide information that is not revealed on axial 2D-CT. ${ }^{25}$ However, the potential risks associated with exposure to radiation are well known, and thus, some authors argue that 3D-CT should not be routinely used in craniosynostosis. ${ }^{9,12,16}$ The results of the current study show that the BB-MRI protocol also provides high-quality 3D-reconstructed skull images and visualization of calvarian sutures and the skull bone structure. Preoperative findings and diagnostic assessment on BB-MRI were consistent with findings from $3 \mathrm{D}-\mathrm{CT}$ in 9 patients with single-suture synostosis. As a limitation of this study, the number of patients in this series is relatively low. However, all images were analyzed by five raters, and all affected synostotic sutures could be identified by each rater from both BB-MRI and 3D-CT images, which increases the power of this study.

Increased ICP is one of the most serious functional concerns in craniosynostosis. The presence of diffuse intracranial impressions (or copper beating) in the inner calvarian surface and erosion of the dorsum sella are typical signs associated with increased ICP. ${ }^{26}$ However, it remains controversial whether mild or localized intracranial impressions represent increased ICP. ${ }^{26}$ To a certain degree, intracranial impressions are considered a "normal" finding in a young child with active growth of the cranium. ${ }^{27}$ In patients with sagittal synostosis, a high incidence of intracranial impressions has been reported ${ }^{28}$ and "diffuse" intracranial impressions are considered a specific sign for increased ICP. ${ }^{26}$ 3D-CT images have been used to identify intracranial impressions in the skull bone. According to 


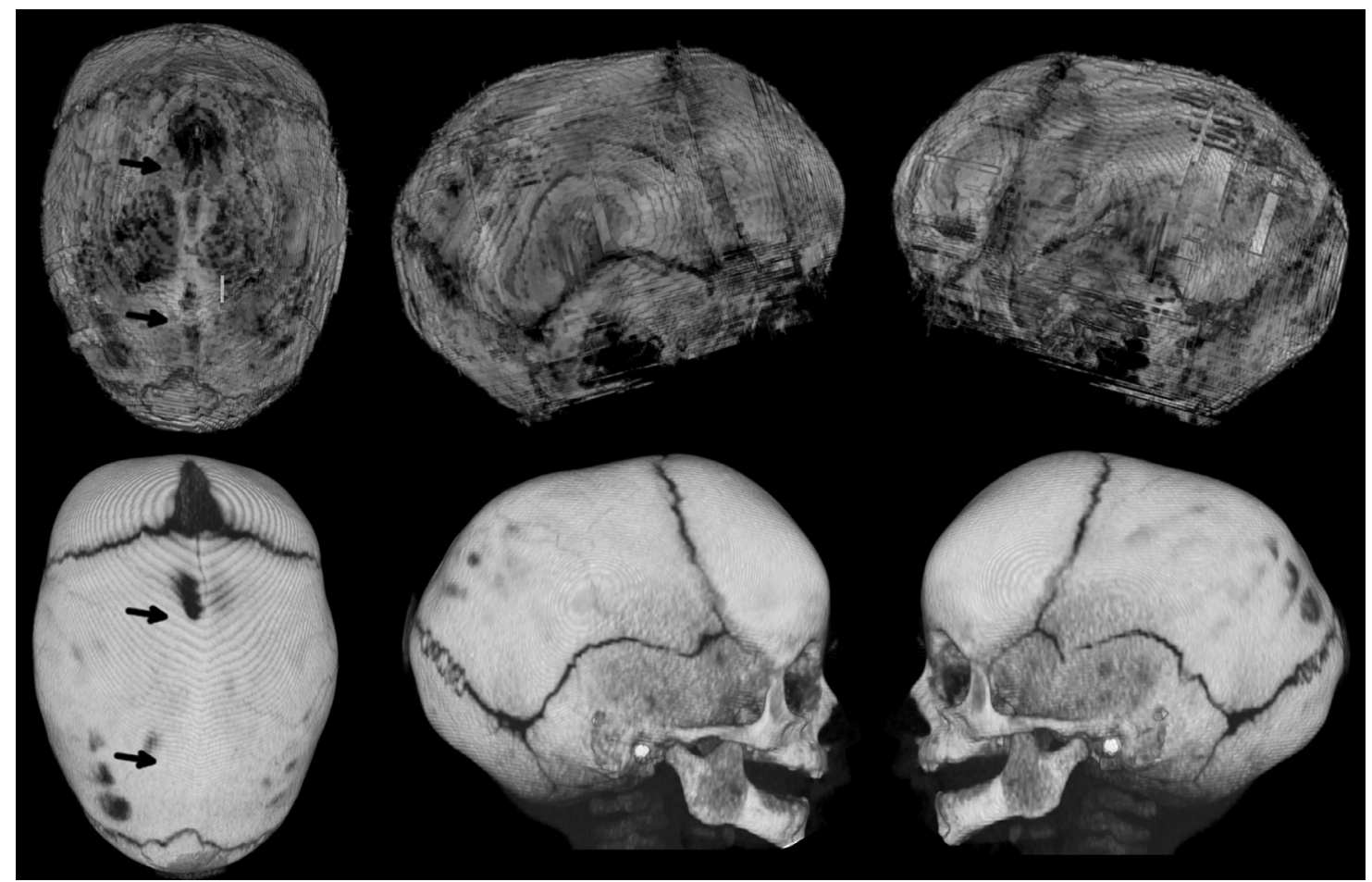

Figure 4 Skull images of patient 3 with sagittal synostosis. Top row, 3D-rendered BB-MRIs at the age of 2.6 months. Bottom row: 3D-rendered CT images at the age of 2.8 months. Imaging was performed in natural sleep after feeding. The arrows point to the location of the fused suture.

the current findings, both $3 \mathrm{D}-\mathrm{CT}$ and $\mathrm{BB}-\mathrm{MRI}$ seem to be subjective methods for evaluating the extent of calvarial impressions. Intrarater reliability for both techniques was relatively low for this purpose. MRI seems to slightly overestimate the extent and severity of the impressions compared to CT, but it is unclear which of the methods better estimate the true intraoperative situation.

We observed that image segmentation of the skull is more difficult in younger patients. The skull of a 6-monthold or younger infant is quite thin and has higher water content, and thus, the bone is not always black. In the current study, the calvarian sutures were visualized from the BB-MRI images in the youngest patients, but the visualization of the bony structures was more accurate in the older patients than in younger patients, i.e., those under 6 months. While MRI eliminates the risks of ionizing radiation, increased examination time, need for anesthesia, lower availability, and cost may be the disadvantages of BB-MRI. Of all patients who receive sedation/anesthesia for diagnostic procedures, the pediatric population represents the highest risk and lowest error tolerance subgroup. ${ }^{29}$ Particularly for infants, the greater anesthesia or sedation time can be considered a potential risk for the developing brains. ${ }^{30}$ The Black Bone sequence itself lasts for approximately $3 \mathrm{~min}$, but if there is a need to image the brain, then the whole protocol will take approximately $15 \mathrm{~min}$. In this study, BB-MRI was performed in natural sleep after feeding for patients under 6 months, and a less motion-sensitive MRI sequence was used. For patients with scaphocephaly, we aimed to perform cranioplasty between the ages of 4 to 6 months; thus, imaging at infant age is indicated, but it is possible without anesthesia.
Cranial ultrasound is another radiation-free modality for the evaluation of cranial sutures that does not require anesthesia. ${ }^{31}$ However, this approach has not yet gained widespread acceptance, perhaps because it is age dependent and children with a positive finding at sonography are still often recommended to undergo CT for more precise preoperative evaluation and surgical planning.

MRI is superior to CT in most cases when evaluating structural brain alterations in children. MRI is routinely used in patients with craniosynostosis and some neurological abbreviations. A coexistent Chiari malformation is found in some children with single suture and frequently with syndromic synostosis. ${ }^{32,33}$ In such instances, a tailored and more posterior cranial remodeling may be indicated. One major advantage of BB-MRI over CT is the avoidance of harmful radiation exposure. ${ }^{12}$ Another advantage is that information of the skull bone, sutures, and brain can be obtained in one imaging session. Of our 9 patients with single-suture synostosis, only one had minor cerebellar germinal matrix hemorrhages considered an incidental finding.

In syndromic craniosynostosis, there is a high risk of concomitant dural venous abnormalities owing to abnormal dural sinus maturation, venous hypertension, and venoocclusive disease. ${ }^{34}$ For these patients, venous imaging is needed for preoperative planning. Knowledge of the exact location, shape, and size of venous sinuses is very useful information for the surgeon in all types of cranial remodeling procedures, including both single-suture and syndromic craniosynostosis. The risk of a venous sinus tear should be minimized while making the bony cuts across the midline. We believe that MRI may have an important 
Table 2 Diagnosis assigned by five different assessors.

\begin{tabular}{|c|c|c|c|c|c|c|c|c|}
\hline \multirow[t]{2}{*}{ ID } & \multirow[t]{2}{*}{ Diagnosis by $\mathrm{CT}$} & \multicolumn{7}{|l|}{ Diagnosis by BB-MRI } \\
\hline & & $\begin{array}{l}\text { Craniofacial } \\
\text { surgeon } 1\end{array}$ & $\begin{array}{l}\text { Craniofacial } \\
\text { surgeon } 2\end{array}$ & Neurosurgeon & Radiologist 1 & $\begin{array}{l}\text { Radiologist 1, 2nd } \\
\text { evaluation }\end{array}$ & Radiologist 2 & $\begin{array}{l}\text { Radiologist 2, 2nd } \\
\text { evaluation }\end{array}$ \\
\hline P1 & $\begin{array}{l}\text { Right coronal } \\
\text { synostosis }\end{array}$ & Right coronal & Right coronal & Right coronal & Right coronal & Right coronal & Right coronal & Right coronal \\
\hline P2 & Sagittal synostosis & Sagittal & Sagittal posterior* & Sagittal & Sagittal & Sagittal & Sagittal & Sagittal \\
\hline P3 & Sagittal synostosis & Sagittal posterior* & Sagittal & Sagittal & Sagittal & Sagittal & Sagittal & Sagittal \\
\hline P4 & Sagittal synostosis & Sagittal & Sagittal & Sagittal & $\begin{array}{l}\text { Sagittal and right } \\
\text { lamboid* }\end{array}$ & $\begin{array}{l}\text { Sagittal, right } \\
\text { lamboid* and } \\
\text { left coronal* }\end{array}$ & Sagittal & $\begin{array}{l}\text { Sagittal and left } \\
\text { coronal* }\end{array}$ \\
\hline P5 & Sagittal synostosis & Sagittal & Sagittal & Sagittal & Sagittal & Sagittal & Sagittal & Sagittal \\
\hline P6 & $\begin{array}{l}\text { Left lamboid } \\
\text { synostosis }\end{array}$ & Left lamboid & Left lamboid & Left lamboid & Left lamboid & Left lamboid & Left lamboid & Left lamboid \\
\hline P7 & $\begin{array}{l}\text { Left lamboid } \\
\text { synostosis }\end{array}$ & Left lamboid & Left lamboid & Left lamboid & Left lamboid & Left lamboid & Left lamboid & Left lamboid \\
\hline P8 & Sagittal synostosis & Sagittal & Sagittal & $\begin{array}{l}\text { Sagittal and right } \\
\text { coronal* }^{*}\end{array}$ & Sagittal & Sagittal & Sagittal & $\begin{array}{l}\text { Sagittal and right } \\
\text { coronal* }^{*}\end{array}$ \\
\hline P9 & Sagittal synostosis & Sagittal & Sagittal & Sagittal & Sagittal & $\begin{array}{l}\text { Sagittal and left } \\
\text { lamboid* }\end{array}$ & Sagittal & Sagittal \\
\hline
\end{tabular}

Table 3 Impressions - CT vs. BB-MRI.

\begin{tabular}{|c|c|c|c|c|c|c|c|c|c|c|c|c|c|c|c|c|c|c|}
\hline & \multicolumn{2}{|l|}{ P1 } & \multicolumn{2}{|l|}{ P2 } & \multicolumn{2}{|l|}{ P3 } & \multicolumn{2}{|l|}{ P4 } & \multicolumn{2}{|l|}{ P5 } & \multicolumn{2}{|l|}{ P6 } & \multicolumn{2}{|l|}{ P7 } & \multicolumn{2}{|l|}{ P8 } & \multicolumn{2}{|l|}{ P9 } \\
\hline & $\mathrm{CT}$ & BB-MRI & CT & BB-MRI & CT & BB-MRI & CT & BB-MRI & $\overline{C T}$ & BB-MRI & CT & BB-MRI & CT & BB-MRI & CT & BB-MRI & СT & BB-MRI \\
\hline Craniofacial surgeon 2 & 021 & 022 & 012 & 012 & 012 & 012 & 112 & 112 & 012 & 012 & - & 012 & 001 & 001 & 012 & 012 & - & 111 \\
\hline Neurosurgeon & 111 & 011 & 001 & 011 & 011 & 011 & 001 & 011 & 010 & 011 & 000 & 011 & 011 & 011 & 010 & 000 & 011 & 000 \\
\hline Radiologist $1^{*}$ & 122 & 122 & 021 & 011 & 022 & 011 & 000 & 010 & 021 & 022 & 000 & 022 & 000 & 011 & 021 & 011 & 011 & 011 \\
\hline Radiologist 2 & 122 & 122 & 011 & 011 & 021 & 011 & 010 & 011 & 021 & 022 & 000 & 022 & 111 & 121 & 011 & 111 & 010 & 111 \\
\hline Radiologist 2* & 122 & 121 & 121 & 011 & 122 & 111 & 011 & 121 & 121 & 022 & - & 022 & 111 & 122 & 021 & 211 & 011 & 111 \\
\hline
\end{tabular}

* Second evaluation. 
role in preventing this major complication. MR venography provides information on the extent of veno-occlusive disease and on the brain. In the future, combining the BB sequence with MRI venography and routine brain MRI will cover all critical aspects in preoperative planning needed for complex syndromic craniosynostosis cases.

In conclusion, preoperative findings and diagnostic assessment on BB-MRI were consistent with the findings on the 3D-CT in 9 patients with single-suture synostosis. Thus, the MRI protocol with the BB-MRI sequence used in this study provides a very promising alternative to $\mathrm{CT}$ when imaging patients with craniosynostosis or any calvarial deformity. This protocol provides information on all critical aspects needed for preoperative planning in patients with craniosynostosis.

\section{Declaration of Competing Interest}

None declared.

\section{CRediT authorship contribution statement}

Anne Saarikko: Conceptualization, Formal analysis, Writing - original draft, Data curation. Eero Mellanen: Writing - original draft, Methodology, Data curation. Linda Kuusela: Formal analysis, Software, Methodology, Data curation. Junnu Leikola: Formal analysis, Writing - review \& editing. Atte Karppinen: Formal analysis, Writing - review $\&$ editing. Taina Autti: Conceptualization, Writing - review \& editing. Pekka Virtanen: Formal analysis, Methodology. Nina Brandstack: Formal analysis, Methodology, Investigation, Writing - review \& editing.

\section{Funding}

None.

\section{Ethical approval}

Ethical approval for the use of BB-MRI was granted by Helsinki University Hospital Research Ethics Committee.

\section{References}

1. Madeline LA, Elster AD. Suture closure in the human chondrocranium: CT assessment. Radiology 1995;196(3):747-56.

2. Persing JA. MOC-PS(SM) CME article: management considerations in the treatment of craniosynostosis. Plast Reconstr Surg 2008;121(4 Suppl):1-11.

3. Albright AL, Byrd RP. Suture pathology in craniosynostosis. J Neurosurg 1981;54(3):384-7.

4. Boulet SL, Rasmussen SA, Honein MA. A population-based study of craniosynostosis in metropolitan atlanta, 1989-2003. Am J Med Genet A 2008;146A(8):984-91.

5. Kweldam CF, van der Vlugt JJ, van der Meulen J. The incidence of craniosynostosis in the netherlands, 1997-2007. J Plast Reconstr Aesth Surg 2010;64(5):583-8.

6. Cornelissen M, Ottelander Bd, Rizodopoulos D, et al. Increase of prevalence of craniosynostosis. J Cranio-Maxillo-Fac Surg 2016(9):1273-9.
7. Selber J, Reid RR, Chike-Obi CJ, et al. The changing epidemiologic spectrum of single-suture synostoses. Plast Reconstr Surg 2008;122(2):527-33.

8. Kirmi O, Lo SJ, Johnson D, Anslow P. Craniosynostosis: a radiological and surgical perspective. Semin Ultrasound CT MRI 2009;30(6):492-512.

9. Fearon JA, Singh DJ, Beals SP, Yu JC. The diagnosis and treatment of single-sutural synostoses: are computed tomographic scans necessary. Plast Reconstr Surg 2007;120(5):1327-31.

10. Badve C, Mallikarjunappa K, Iyer R, Ishak G, Khanna P. Craniosynostosis: imaging review and primer on computed tomography. Pediatr Radiol 2013;43(6):728-42.

11. Nagaraja S, Anslow P, Winter B. Craniosynostosis. Clin Radiol 2012;68(3):284-92.

12. Frush DP, Donnelly LF, Rosen NS. Computed tomography and radiation risks: what pediatric health care providers should know. Pediatrics 2003;112(4):951-7.

13. Pearce MS, Salotti JA, Little MP, et al. Radiation exposure from CT scans in childhood and subsequent risk of leukaemia and brain tumours: a retrospective cohort study. Lancet 2012;380(9840):499-505.

14. Cardis E, Vrijheid M, Blettner M, et al. Risk of cancer after low doses of ionising radiation: retrospective cohort study in 15 countries. BMJ 2005;331(7508):77-80.

15. Kaasalainen $T$, Palmu K, Lampinen A, et al. Limiting $C T$ radiation dose in children with craniosynostosis: phantom study using model-based iterative reconstruction. Pediatr Radiol 2015;45(10):1544-53.

16. Schweitzer T, Böhm H, Meyer-Marcotty P, Collmann H, Ernestus R-, Krauß J. Avoiding CT scans in children with single-suture craniosynostosis. Childs Nerv Syst 2012;28(7):1077-82.

17. Brenner DJ, Hall EJ. Computed tomography - an increasing source of radiation exposure. $N$ Engl J Med 2007;357(22):2277-84.

18. Eley KA, Sheerin F, Taylor N, Watt-Smith SR, Golding SJ. Identification of normal cranial sutures in infants on routine magnetic resonance imaging. J Craniofac Surg 2013;24(1):317-20.

19. Eley KA, Watt-Smith SR, Sheerin F, Golding SJ. "Black bone" MRI: a potential alternative to $C T$ with three-dimensional reconstruction of the craniofacial skeleton in the diagnosis of craniosynostosis. Eur Radiol 2014;24(10):2417.

20. Eley KA, Watt-Smith SR, Golding SJ. "Black bone" MRI: a potential alternative to $C T$ when imaging the head and neck: report of eight clinical cases and review of the oxford experience. $\mathrm{Br}$ J Radiol 2012;85(1019):1457.

21. Eley KA, McIntyre AG, Watt-Smith SR, Golding SJ. "Black bone" MRI: a partial flip angle technique for radiation reduction in craniofacial imaging. Br J Radiol 2012;85(1011):272-8.

22. Kuusela L, Hukki A, Brandstack N, Autti T, Leikola J, Saarikko A. Use of black-bone MRI in the diagnosis of the patients with posterior plagiocephaly. Childs Nerv Syst 2018;34(7):1383-9.

23. Chandarana H, Block TK, Rosenkrantz AB, et al. Free-breathing radial 3D fat-suppressed $\mathrm{T} 1$-weighted gradient echo sequence: a viable alternative for contrast-enhanced liver imaging in patients unable to suspend respiration. Invest Radiol 2011;46(10):648-53.

24. Ahmed MN, Yamany SM, Mohamed NA, Farag AA. A modified fuzzy C-means algorithm for MRI bias field estimation and adaptive segmentation. In: Taylor C, Colchester A, editors. Medical image computing and computer-assisted intervention - MICCAl'99. Berlin Heidelberg: Springer-Verlag; 1999. p. 72-81.

25. Vannier MW, Hildebolt CF, Marsh JL, et al. Craniosynostosis: diagnostic value of three-dimensional CT reconstruction. Radiology 1989;173(3):669-73.

26. Tuite GF, Evanson J, Chong WK, et al. The beaten copper cranium: a correlation between intracranial pressure, cranial radiographs, and computed tomographic scans in children with craniosynostosis. Neurosurgery 1996;39(4):691-9. 
27. du Boulay G. The significance of digital impressions in children's skulls. Acta Radiol 1956;46(1-2):112-22.

28. Agrawal D, Steinbok $P$, Cochrane DD. Significance of beaten copper appearance on skull radiographs in children with isolated sagittal synostosis. Childs Nerv Syst 2007;23(12):1467-70.

29. Cravero JP, Blike GT, Beach M, et al. Incidence and nature of adverse events during pediatric sedation/anesthesia for procedures outside the operating room: report from the pediatric sedation research consortium. Pediatrics 2006;118(3):1087-96.

30. Andropoulos DB, Greene MF. Anesthesia and developing brains - implications of the FDA warning. N Engl J Med 2017;376(10):905-7.

31. Hall KM, Besachio DA, Moore MD, Mora AJ, Carter WR. Effectiveness of screening for craniosynostosis with ultrasound: a retrospective review. Pediatr Radiol 2017;47(5):606-12.
32. Leikola J, Koljonen V, Valanne L, Hukki J. The incidence of chiari malformation in nonsyndromic, single suture craniosynostosis. Childs Nerv Syst 2010;26(6):771-4.

33. Hukki A, Koljonen V, Karppinen A, Valanne L, Leikola J. Brain anomalies in 121 children with non-syndromic single suture craniosynostosis by MR imaging. Eur J Paediatr Neurol 2012;16(6):671-5.

34. Sandberg DI, Navarro R, Blanch J, Ragheb J. Anomalous venous drainage preventing safe posterior fossa decompression in patients with chiari malformation type I and multisutural craniosynostosis. report of two cases and review of the literature. J Neurosurg 2007; 106(6 Suppl):490-4. 